B.-Q. $\mathrm{Li}$

KODAI MATH. J.

11 (1988), 287-294

\title{
ON THE QUANTITY $\delta_{s}(g(z), f)$ OF GAPPY ENTIRE FUNCTIONS
}

\author{
BY BAO-QIN LI
}

\section{Introduction.}

Let $f(z)=\sum_{n=0}^{\infty} a_{n} z^{n}$ be a transcendental entire function. We denote by $\Lambda_{f}=$ $\left\{\lambda_{k}\right\}, M_{f}=\left\{u_{k}\right\}, k=1,2, \cdots$ the sequences of exponents $n$ for which $a_{n} \neq 0$ and $a_{n}=0$ respectively, arranged in increasing order. Also let $g(z)$ be an arbitrary meromorphic function in the plane growing slowly compared with the function $f(z)$, i. e., $T(r, g)=o\{T(r, f)\}$ as $r \rightarrow \infty$.

If $f(z)$ has finite order we define

$$
\delta_{s}(g(z), f)=1-\varliminf_{r \rightarrow \infty} \frac{N\left(r, \frac{1}{f-g(z)}\right)}{T(r, f)} .
$$

If $f(z)$ has infinite order, let $E$ be any set in $(1, \infty)$ having finite length. We define

$$
\delta_{s}(g(z), f)=1-\sup _{E} \lim _{r \rightarrow \infty, r \notin E} \frac{N\left(r, \frac{1}{f-g(z)}\right)}{T(r, f)}=\inf _{E} \varlimsup_{r \rightarrow \infty, r \notin E} \frac{m\left(r, \frac{1}{f-g(z)}\right)}{T(r, f)} .
$$

Obviously

$$
\delta(g(z), f)=1-\varlimsup_{r \rightarrow \infty} \frac{N\left(r, \frac{1}{f-g(z)}\right)}{T(r, f)} \leqq \delta_{s}(g(z), f) .
$$

In $[1]$, we obtained the following

THEOREM A. Let $d n$ be the highest common factor of all the numbers $\lambda_{m+1}-\lambda_{m}$ for $m \geqq n$ and suppose that

Then

$$
d_{n} \longrightarrow \infty \quad \text { as } n \rightarrow \infty \text {. }
$$

$$
\delta_{s}(g(z), f)=0
$$

for every entire function $g(z)$ satisfying $T(r, g)=o\{T(r, f)\}$ as $r \rightarrow \infty$.

Received January 19, 1988 
In this paper we further prove that (1.2) is also valid for every meromorphic function $g(z)$ satisfying $T(r, g)=o\{T(r, f)\}$ as $r \rightarrow \infty$. That is, we shall prove

THEOREM 1. With the hypotheses of Theorem A, we have

$$
\delta_{s}(g(z), f)=0
$$

for every meromorphic function $g(z)$ satisfying $T(r, g)=o\{T(r, f)\}$ as $r \rightarrow \infty$.

From (1.1), we see that $\Lambda_{f}$ is very 'thin' in a sense. For the gappy entire function $f(z)$ with 'thickish' $\Lambda_{f}$, we have

THEOREM 2. Suppose that $b(\geqq 0), d(\geqq 2)$ are integers such that $b+k d \in M_{f}$ for $k \geqq 1$. Then for every meromorphic function $g(z)$ satisfying $T(r, g)=o\{T(r, f)\}$ as $r \rightarrow \infty$, we have

$$
\delta_{s}(g(z), f) \leqq 1-\frac{1}{d}
$$

except possibly if, for $0<|z|<\varepsilon, g(z)=\frac{1}{z^{m}} \sum_{n=0}^{\infty} C n z^{n}$ and $C_{b+m}=a_{b}$, where $m(\geqq 0)$ is an integer and $\varepsilon$ is a positive number.

Remark 1. (1.3) can break down if $g(z)=\frac{1}{z^{m}} \sum_{n=0}^{\infty} C n z^{n}$ and $C_{b+m}=a_{b}$. For

$$
f(z)=\frac{1}{6}+\sum_{n=1}^{\infty} \frac{z^{2 n}}{(n+3) !}
$$

satisfies the hypotheses of Theorem 2 with $b=1$ and $d=2$. But for $g(z)=$ $-\left(1 / z^{6}\right)\left(1+z^{2}+z^{4} / 2\right)$, with $m=6$ and $C_{b+m}=a_{b}=0$, we have

$$
f(z)-g(z)=\frac{1}{z^{6}}\left(1+z^{2}+\frac{z^{4}}{2}+\frac{z^{6}}{6}+\sum_{n=1}^{\infty} \frac{z^{2(n+3)}}{(n+3) !}\right)=\frac{e^{z^{2}}}{z^{6}}
$$

and so

Also for

$$
\delta_{s}(g(z), f)=1 \text {. }
$$

$$
f(z)=z+e^{z^{2}}
$$

satisfies the hypotheses of Theorem 2 with $b=1$ and $d=2$. But for $g(z)=z$, with $m=0$ and $C_{b+m}=1=a_{b}$, we have

$$
\delta_{s}(z, f)=1 \text {. }
$$

Remark 2. The inequality (1.3) is sharp. For $f(z)=-\frac{\sqrt{\pi}}{2}+\int_{0}^{z} e^{-t^{2}} d t$ satisfies the hypotheses of Theorem 2 with $b=0$ and $d=2$. Hence we have $\delta_{s}(0, f) \leqq$ $1-1 / 2=1 / 2$. Also as is shown by Nevenlinna [5], we have $\delta(0, f)=1 / 2$. Thus 
$\delta_{s}(0, f) \geqq \delta(0, f)=1 / 2$. Therefere $\delta_{s}(0, f)=1 / 2$.

As a consequence of Theorem 2, we have

COROLlARY 1. Suppose that $b(>0), d(\geqq 2)$ are integers such that $b \in \Lambda_{f}$ but $b+k d \in M_{f}$ for $k \geqq 1$. Then

$$
\sum_{\alpha \neq \infty} \delta(a, f) \leqq 1-\frac{1}{d} .
$$

Remark 3. (1.4) is obviously better than the corresponding result of Hayman in $[3$, p. 330$]: \sum_{a \neq \infty} \delta(a, f) \leqq 1-\frac{1}{d(d-1)}$ with the hypotheses of Corollary 1 .

\section{Proof of Theorems.}

From now on we denote by $S(r, F)$ any term which satisfies $S(r, F)=$ $o\{T(r, F)\}$ as $r \notin E, r \rightarrow \infty$, where $E$ is a set in $(1,+\infty)$ having finite length and in particular $E$ is an empty set if $F(z)$ is of finite order.

In proving our theorems, we shall quote the following lemmas.

LEMMA 1. [2]: If $F(z)$ is a transcendental entire function and $g_{1}(z), g_{2}(z)$, $\cdots, g_{q}(z)$ are distinct meromorphic functions satisfying $T\left(r, g_{j}\right)=o\{T(r, F)\}$ as $r \rightarrow \infty(j=1,2, \cdots, q)$, then

$$
\sum_{j=1}^{q} m\left(r, \frac{1}{F-g_{j}(z)} \leqq T(r, F)+S(r, F) .\right.
$$

LEMMA 2 [4]: If $a_{1}, \cdots, a_{q}$ are distinct finite complex numbers, then whenever $F(z)$ is transcendental entire function we have

$$
\sum_{\jmath=1}^{q} m\left(r, \frac{1}{F-a_{\jmath}}\right) \leqq m\left(r, \frac{1}{F^{\prime}}\right)+S(r, F) .
$$

Proof of Theorem 1.

By our Theorem A, we may assume that $g(z)$ is a meromorphic function which has at least a pole and satisfies

$$
T(r, g)=o\{T(r, f)\} \quad \text { as } r \rightarrow \infty
$$

Let's discuss two cases separately.

Case (a). $g(z)$ has a pole $Z_{o} \neq 0$.

Let $q(\geqq 2)$ be an arbitrary integer. We write

$$
\begin{aligned}
& W n=\exp \left(\frac{2 \pi i}{d_{n}}\right), \\
& P(z)=\sum_{v=0}^{n-1} a_{\lambda v} z^{\lambda v},
\end{aligned}
$$




$$
F(z)=\sum_{v=n}^{\infty} a_{\lambda v} z^{\lambda v}
$$

Obviously, $\lim _{n \rightarrow \infty}(W n)^{j} Z o=Z o(1 \leqq j \leqq q)$. Hence there exists a larger $n$ such that $(W n)^{j} Z_{o}(1 \leqq j \leqq q)$ is not a pole of $g(z)$.

Noticing $d_{n} \mid\left(\lambda_{m+1}-\lambda_{m}\right)$ for $m \geqq n$, we have

$$
d_{n} \mid\left(\lambda_{v}-\lambda_{n}\right) \quad \text { for } v \geqq n,
$$

where " $a \mid b$ " denotes that $a$ is a factor of $b$. Thus for $1 \leqq j \leqq q$ we have, in view of (2.2) and (2.3),

$$
\begin{aligned}
f\left(W_{n}^{j} z\right)-g\left(W_{n}^{j} z\right) & =p\left(W_{n}^{j} z\right)-g\left(W_{n}^{j} z\right)+F\left(W_{n}^{j} z\right) \\
& =p\left(W_{n}^{j} z\right)-g\left(W_{n}^{j} z\right)+W_{n}^{j \lambda n} F(z) \\
& =W_{n}^{j \lambda n}\left\{F(z)-W_{n}^{-j \lambda n}\left(g\left(W_{n}^{j} z\right)-p\left(w_{n}^{j} z\right)\right)\right\} \\
& =W_{n}^{j \lambda n}\left\{F(z)-g_{j}(z)\right\},
\end{aligned}
$$

say, where $g_{j}(z)=W_{n}^{-j \lambda n}\left(g\left(W_{n}^{j} z\right)-p\left(W_{n}^{j} z\right)\right)$.

Clearly, by (2.1) we have

$$
T\left(r, g_{j}(z)\right)=o\{T(r, f)\} \quad \text { as } r \rightarrow \infty .
$$

We assert that $g_{1}(z), g_{2}(z), \cdots, g_{q}(z)$ are dist.nct from each other. Otherwise, there must exist $i, j(1 \leqq i<j \leqq q)$ such that

$$
\Delta_{i j}(z)=W n^{-j \lambda n}\left(g\left(W n^{j} z\right)-p\left(W n^{j} z\right)\right)-W n^{-i \lambda n}\left(g\left(W n^{i} z\right)-p\left(W n^{i} z\right)\right) \equiv 0 .
$$

But by the definition of the integer $n$ we easily conclude that

$$
\Delta_{i j}\left(W n^{-i} z o\right)=W n^{-j \lambda n}\left(g\left(W n^{j-i} z o\right)-p\left(W n^{j-i} z o\right)-W n^{i \lambda n}(g(z o)-p(z o))=\infty .\right.
$$

This contradicts (2.6).

Now using Lemma 1 to our functions $g_{1}(z), g_{2}(z), \cdots, g_{q}(z)$ and $F(z)$ and noticing (2.4) and (2.5), we deduce that

$$
\sum_{j=1}^{q} m\left(r, \frac{1}{F-g_{\jmath}(z)}\right) \leqq T(r, F)+S(r, F)
$$

and so that

$$
\begin{aligned}
q m\left(r, \frac{1}{f-g(z)}\right) & \leqq T(r, F)+S(r, F) \\
& \leqq T(r, f)+S(r, f)
\end{aligned}
$$

Thus we have

$$
\delta_{s}(g(z), f) \leqq \frac{1}{q} .
$$


But $q$ can be assumed any large. Hence we obtain

$$
\delta_{s}(g(z), f)=0 .
$$

Case (b). $\quad Z o=0$ is an only pole of $g(z)$.

It is easily seen that there exists an integer $m \geqq 1$ such that

$$
g(z)=\frac{1}{z^{m}} g^{*}(z),
$$

where $g^{*}(z)$ is an entire function satisfying

$$
T\left(r, g^{*}(z)\right)=o\{T(r, f)\} \quad \text { as } r \rightarrow \infty .
$$

we set

$$
\begin{aligned}
f^{*}(z) & =z^{m} f(z) \\
& =z^{m} \sum_{n=1}^{\infty} a n z^{\lambda_{n}}=\sum_{n=1}^{\infty} a_{\lambda_{n}} z^{\eta_{n}},
\end{aligned}
$$

say, where $\eta_{n}=\lambda_{n}+m$.

By (2.7) we have

$$
T\left(r, g^{*}(z)\right)=o\left\{T\left(r, f^{*}\right)\right\} \quad \text { as } r \rightarrow \infty .
$$

Let $d n^{*}$ be the highest common factor of all the numbers $\eta_{s+1}-\eta_{s}$ for $s \geqq n$. Apparently we have

$$
d n^{*} \longrightarrow \infty \quad \text { as } n \rightarrow \infty \text {. }
$$

Using Theorem A to our functions $f^{*}(z)$ and $g^{*}(z)$ and noticing (2.8) and (2.9), we get

But

$$
\delta_{s}\left(g^{*}(z), f^{*}(z)\right)=0
$$

$$
f(z)-g(z)=\frac{1}{z^{m}}(f *(z)-g *(z))
$$

Therefore

$$
\delta s(g(z), f)=\delta s\left(g^{*}(z), f^{*}\right)=0 .
$$

This completes the proof of Theorem 1 .

Proof of Theorem 2.

Suppose that $g(z)$ is any meromorphic function satisfying

we write

$$
T(r, g)=o\{T(r, f)\} \quad \text { as } r \rightarrow \infty
$$

$$
\begin{gathered}
W=\exp \left(\frac{2 \pi i}{d}\right), \\
h_{v}(z)=W^{-v b}\left\{f\left(W^{v} z\right)-g\left(W^{v} z\right)\right\} \quad(1 \leqq v \leqq d), \\
h(z)=\sum_{v=1}^{d} h_{v}(z) .
\end{gathered}
$$


We then have, for any integer $n(\geqq 0)$, that $\sum_{v=1}^{d} W^{v(n-b)}=d$ if $d \mid(n-b)$ and $\sum_{v=1}^{d} W^{v(n-b)}=0$ if $d \chi(n-b)$.

So by $b+k d \in M_{f}$ for $k \geqq 1$ we deduce that

$$
\begin{aligned}
h(z) & =\sum_{v=1}^{d} h_{v}(z) \\
& =\sum_{v=1}^{d} W^{-v b} \sum_{n=0}^{\infty} a_{n} W^{v n} z^{n}-\sum_{v=1}^{d} W^{-v b} g\left(W^{v} z\right) \\
& =d \sum_{k=k_{0}}^{0} a_{b+k d} z^{b+k d}-\sum_{v=1}^{d} W^{-v b} g\left(W^{v} z\right),
\end{aligned}
$$

where $k o$ is the minimum of integers $k(-\infty<k \leqq 0)$ satisfying $b+k d \geqq 0$.

By (2.10), it is easily deduced that

$$
T(r, h(z))=o\{T(r, f)\} \quad \text { as } r \rightarrow \infty .
$$

We claim that

$$
h(z) \not \equiv 0,
$$

if the exceptional case mentioned in Theorem 2 doesn't occur.

In fact, we may write

$$
g(z)=\frac{1}{z^{m}} \sum_{n=0}^{\infty} C n z^{n} \quad \text { as } 0<|z|<\varepsilon
$$

where $m(\geqq 0)$ is an integer and $\varepsilon$ is a small positive number.

Hence for $0<|z|<\varepsilon$ we have

$$
\begin{aligned}
h(z) & =d \sum_{k=k_{0}}^{0} a_{b+k d} z^{b+k d}-\sum_{n=0}^{\infty} C n z^{n-m} \sum_{v=1}^{d} W^{v(n-m-b)} \\
& =d\left(\sum_{k=k_{0}}^{0} a_{b+k d} z^{b+k d}-\sum_{k=k_{1}}^{\infty} C m+b+k d z^{b+k d}\right),
\end{aligned}
$$

where $k_{1}$ is the minimum of integer $k(-\infty<k \leqq 0)$ satisfying $m+b+k d \geqq 0$.

In (2.15), if $z=0$ is a pole of $h(z)$, then (2.14) is alreadly correct. Or $h(z)$ is holomorphic in $\{|z|<\varepsilon\}$ and (2.15) is its power-series expansion in $|z|<\varepsilon$. But the coefficient of $z^{b}$ in $h(z)$ is $d\left(a_{b}-C_{m+b}\right) \neq 0$. Thus (2.14) is also correct.

Next we assume without loss of generality that $h_{1}(z), h_{2}(z), \cdots, h_{t}(z)(t \leqq d)$ are linearly independent. Then there exist complex numbers $e_{1}, e_{2}, \cdots, e_{f}$ such that

$$
\begin{aligned}
h(z) & =e_{1} h_{1}(z)+e_{2} h_{2}(z)+\cdots+e_{t} h_{t}(z) \\
& =H_{1}(z)+H_{2}(z)+\cdots+H_{t}(z),
\end{aligned}
$$

say, where $H j(z)=e j h j(z)(1 \leqq j \leqq t)$.

Also we may assume that $e_{j} \neq 0$ for $1 \leqq j \leqq t$, which doesn't, lose generality. 
Hence if we set

then we have $\Delta \not \equiv 0$.

$$
\Delta=\left|\begin{array}{cccc}
H_{1}(z) & H_{2}(z) & \cdots & H_{t}(z) \\
H_{1}{ }^{\prime}(z) & H_{2}{ }^{\prime}(z) & \cdots & H_{t}{ }^{\prime}(z) \\
\cdots & \cdots & \cdots & \cdots \\
H_{1}{ }^{(t-1)}(z) & H_{2}{ }^{(t-1)}(z) & \cdots & H_{t}^{(t-1)}(z)
\end{array}\right|,
$$

By (2.16), we conclude that

$$
\begin{aligned}
\Delta & =\left|\begin{array}{cccc}
h(z) & H_{2}(z) & \cdots & H_{t}(z) \\
h^{\prime}(z) & H_{2}{ }^{\prime}(z) & \cdots & H_{t}{ }^{\prime}(z) \\
\cdots & \cdots & \cdots & \cdots \\
h^{(t-1)}(z) & H_{2}{ }^{(t-1)}(z) & \cdots & H_{t}{ }^{(t-1)}(z)
\end{array}\right| \\
& =h(z) \Delta_{0}(z)+h^{\prime} \Delta_{1}(z)+\cdots+h^{(t-1)}(z) \Delta_{t-1}(z),
\end{aligned}
$$

where $\Delta_{j}(z)(0 \leqq j \leqq t-1)$ is the algebraic complement with respect to $h^{(j)}(z)$ $\left(h^{(0)}(z) \equiv h(z)\right)$.

It is well known that $m\left(r, F^{(k)} / F\right)=S(r, F)$ for $k \geqq 1$ whenever $F(z)$ (声constant) is a meromorphic function [4]. Hence we deduce that, in view of (2.11), (2.17) and (2.13),

$$
\begin{aligned}
T(r, f) \leqq & T\left(r, H_{1}(z)\right)+o\{T(r, f)\} \\
\leqq & N\left(r, H_{1}(z)\right)+m\left(r, \frac{\sum_{v=0}^{t-1} h^{(v)} \Delta_{v}}{\prod_{v=2}^{t} H_{v}}\right)+m\left(r, \frac{\prod_{v=1}^{t} H_{v}}{\Delta}\right)+o\{T(r, f)\} \\
\leqq & N\left(r, H_{1}(z)\right)+\sum_{v=0}^{t-1} m\left(r, h^{(v)}\right)+\sum_{v=0}^{t-1} m\left(r, \frac{\Delta_{v}}{\prod_{j=2}^{t} H_{j}}\right)+m\left(r, \frac{\Delta}{\prod_{v=1}^{t} H_{v}}\right) \\
& +N\left(r, \frac{\Delta}{\prod_{v=1}^{t} H_{v}}\right)+o\{T(r, f)\} \\
\leqq & N\left(r, H_{1}(z)\right)+\sum_{v=0}^{t-1} m\left(r, \frac{h^{(v)}}{h}\right)+t m(r, h)+\sum_{v=0}^{t-1} m\left(r, \frac{\Delta_{v}}{\prod_{j=2}^{t} H_{j}}\right) \\
& +m\left(r, \frac{\Delta}{\prod_{v=1}^{t} H_{v}}\right)+N(r, \Delta)+\sum_{v=1}^{t} N\left(r, \frac{1}{H_{v}}\right)+o\{T(r, f)\} \\
\leqq & o\{T(r, f)\}+S(r, h)+o\{T(r, f)\}+\sum_{j=2}^{t} S\left(r, H_{j}\right)+\sum_{j=1}^{t} S\left(r, H_{j}\right) \\
& +o\{T(r, f)\}+t N\left(r, \frac{1}{f-g(z)}\right)+o\{T(r, f)\}
\end{aligned}
$$




$$
\leqq t N\left(r, \frac{1}{f-g(z)}\right)+S(r, f) .
$$

That is,

$$
T(r, f) \leqq t N\left(r, \frac{1}{f-g(z)}\right)+S(r, f) .
$$

Therefore we conclude that

$$
\delta_{s}(g(z), f) \leqq 1-\frac{1}{t} \leqq 1-\frac{1}{d} .
$$

The proof of Theorem 2 is thus completed.

Using Theorem 2 to the functions $f^{\prime}(z)$ and $g(z)=0$ and Lemma 2 to the function $f$, we easily get the conclusion of Corollary 1 . We here omit its details.

\section{REFERENCES}

[1] BAo-QIn Li, Remarks on a result of Hayman, Kodai Math. J., 11 (1988), 32-37.

[2] C. T. ChuAng, Une generalisation d'une inegalite de Nevanlinna, Sci. Sinica, 13 (1964), 887-895.

[3] W. K. Hayman, Value distribution and A.P. gaps, J. London Math. Soc. (2), 28 (1983), 327-338.

[4] W.K. HAYMAN, Meromorphic functions, Oxford, 1964.

[5] R. NevanlinNa, Le theoreme de picard-Borel et la theorie des functions meromorphes, Gauthier-Villars, Paris, 1929.

Department of Mathematics

Yangzhou Teachers College

JiAng Su, China 